\title{
Thermodiffusion of the tetrahydronaphthalene and dodecane mixture under high pressure and in porous medium
}

\author{
Cédric Giraudet ${ }^{\mathrm{a}}$, Fabrizio Croccolo ${ }^{\mathrm{b}}$, Guillaume Galliero ${ }^{\mathrm{a}}$, Gilles Pijaudier-Cabot ${ }^{\mathrm{a}}$, \\ Stefan Van Vaerenbergh ${ }^{c}$, M. Ziad Saghir ${ }^{\mathrm{d}}$, François Montel ${ }^{\mathrm{e}}$, Henri Bataller ${ }^{\mathrm{a}, *}$ \\ a Laboratoire des Fluides Complexes et leurs Réservoirs, UMR 5150, Université de Pau et des Pays de l'Adour, BP 1155, 64013 Pau cedex, France \\ ${ }^{\mathrm{b}}$ Physics Department, University of Fribourg, chemin du Musée 3, 1700 Fribourg, Switzerland \\ ${ }^{c}$ Microgravity Research Center, Université Libre de Bruxelles, CP165/62, 1150 Bruxelles, Belgium \\ ${ }^{\mathrm{d}}$ Ryerson University, 350 Victoria St, Toronto, ONT, M5B 2K3, Canada \\ e Total SA, avenue Larribau, 64018 Pau cedex, France
}

\begin{abstract}
A thermodiffusion cell is used in order to perform Soret experiments on binary mixtures at high pressure and in the presence of a porous medium. The cell is validated at atmospheric pressure with toluene/hexane and the tetrahydronaphthalene/dodecane mixtures. The mass separation follows a diffusive behaviour when the cell is filled with a porous medium. At least three times the relaxation time is needed to have a good estimation of the Soret coefficients. From the transient state of the mass separation and using accepted values of the diffusion coefficient, the tortuosity of the porous medium was evaluated, too. Finally, experiments at high pressure were performed with the tetrahydronaphthalene/dodecane system. In these experiments, decreases of the Soret coefficient and of the tortuosity of the porous medium were measured as a function of the pressure.
\end{abstract}

\section{Introduction}

The presence of a temperature gradient in a fluid mixture generally induces mass flow, thus creating concentration gradients in the mixture. This process is known as the Ludwig-Soret effect [1,2]. In a binary mixture, the amplitude of this effect is characterized by the Soret coefficient $S_{T}$, which relates the concentration gradient to the applied temperature gradient at steady-state conditions:

$$
S_{T}=-\frac{1}{c_{0}\left(1-c_{0}\right)} \frac{\nabla c}{\nabla T}
$$

Here $T$ is the temperature, $c$ the mass fraction of the densest component, $c_{0}$ its initial concentration, and $\nabla$ indicates the gradient. The Soret coefficient as defined by Eq. (1) is conventionally related to component 1 [3] and has a positive (respectively, negative) sign when component 1 migrates to the cold (warm) side of the containing cell. In a binary mixture, the Soret coefficient can also be expressed as $S_{T}=D_{T} / D$, where $D_{T}$ is the thermal diffusion coefficient and $D$ is the molecular diffusion one [4].

\footnotetext{
* Corresponding author.

E-mail address: henri.bataller@univ-pau.fr (H. Bataller).
} 
Transport phenomena in porous media have received considerable attention due to the increasing interest in geothermal processes, chemical catalytic reactors, waste storage (especially geological or ocean storage of carbon dioxide), etc. Among others, the oil industry has shown an increasing interest in studying diffusion phenomena [5]. Since conditions under which the crude oil is found underground imply high pressure (HP) and diffusion in porous medium, it is important to analyze the influence of the pressure and the interaction with a porous medium on the transport properties of dense mixtures. This is done in order to improve the description of the initial distribution of components within a reservoir [6,7]. Nevertheless, thermal diffusion data under reservoir conditions, i.e. at high pressures, are very limited and not very recent $[8,9]$. The most recent reference one may cite are the high-pressure measurements of the thermal diffusion coefficients made with a thermogravitational column [10]. This lack of HP measurements has led to the development of the SCCO project (ESA + Total) [11,12]. The main objective of this project was to perform microgravity measurements of thermal diffusion of various mixtures under HP conditions. Additionally, in the frame of this project, a ground HP experimental device, to measure thermal diffusion and molecular diffusion in binary mixtures, has been designed. The HP thermodiffusion cell is filled with a porous media in order to avoid convection in the fluid and to simulate reservoirs conditions. In precedent works we have used the cell to measure the concentration derivative of the refractive index of a binary mixture $(\partial n / \partial c)_{p, T}$ at high pressure and the temperature derivative of the refractive index of a binary mixture $(\partial n / \partial T)_{p, T}[13-15]$. As a further step, in this paper we report how to obtain molecular diffusion and Soret coefficients of binary mixtures.

The remainder of this paper is organized as follows: in Section 2 the experimental set-up is described; in Section 3 the experimental procedure is exposed; in Section 4 details are provided about the algorithm, allowing us to get the molecular diffusion and Soret coefficients values; in Section 5 experimental results are presented and compared with previous experimental works. Conclusions are provided in Section 6.

\section{Experimental set-up}

The experimental apparatus has been described in more details in previous works [13,15]. The high-pressure cell consists of a stainless steel cylinder with two water circuits at its top and bottom. Pure water coming from two distinct thermostatic baths circulates through these loops to keep the top and bottom of the cell at two distinct temperatures with a stability of about $\pm 0.1^{\circ} \mathrm{C}$ over many days. Most of the interior volume of the cell is filled with a porous medium consisting of a monolithic silica cylinder of the same diameter as the cell interior. At the two extremities of the porous medium, two free volumes, which we refer to as dead volumes (DVs) allow refractive index measurements by means of interferometry. The optical access to the two DVs is provided by two opposing sapphire windows for each DV, letting a laser beam pass through the fluid perpendicular to the cell axis. As reported elsewhere [13], the value of the optical path has been obtained from the analysis of experimental data taken with pure toluene, hexane and water, and it is $d^{*}=21.5 \pm 0.3 \mathrm{~mm}$. The cell is designed to maintain a liquid mixture in a pressure range between 0.1 and $100 \mathrm{MPa}$ and in a temperature range between 5 and $40^{\circ} \mathrm{C}$. The cell is externally covered with a ceramic insulator, in order to limit heat transfer between the cell walls and the environment.

The cell is filled by means of a filling system consisting of a rotary vacuum pump able to evacuate most of the air from the cell before filling operations, a fluid vessel at atmospheric pressure, a manual volumetric pump, and a number of valves used to facilitate the procedure. After a low vacuum is made inside the cell, the mixture to be studied is transferred into the cell by letting it enter from its bottom side. In this phase, visual inspection through the sapphire windows is needed to check bubble presence. The cell is then abundantly fluxed with about $100 \mathrm{ml}$ of the fluid mixture. At the end of the procedure, a valve at the end of the circuit is closed and the volumetric pump is operated to modify the liquid pressure within the cell and perform the experimental runs. A manometer (Keller, PAA-33X/80794, pressure range: 0.1-100 MPa, precision $\pm 0.03 \mathrm{MPa}$ ) is connected between the volumetric pump and the cell in order to constantly check the pressure of the fluid mixture [13]. At the top and bottom of the cell, two K-type thermocouples are positioned within the two DVs in contact with the liquid.

The cell is inserted in a Mach-Zehnder interferometer, each half of the laser beam crossing one DV [15]. The light source consists in a He-Ne laser (Melles Griot, 25 LHP 151-230) operating at a wavelength of $\lambda_{0}=632.8 \mathrm{~nm}$. The beam is deflected by a metallic mirror and is made divergent by means of a positive lens $(f=2 \mathrm{~cm})$. A $50 / 50$ beam splitter further divides the beam into two beams of equal intensity, each beam crossing one DV in order to measure the refractive index difference between the two DVs. After the beam of the bottom is bent by a mirror and finally both beams overlap at a second 50/50 beam splitter and eventually propagate to the CCD camera (Cohu, 7712-3000) after being captured by a microscope objective.

All our tests have been made with the cell in vertical position while heating from above; this allows having a stable configuration from the thermal and composition points of view, at least for all samples with positive Soret coefficient. The entire optical set-up is mounted on an optical table.

\section{Experimental procedure}

The procedure to extract the refractive index variations within the cell has already been described in [13]. The data analysis in the present case is somewhat different and it is described in the following. 
Initially, the two thermoregulated baths are set to the same temperature (mean temperature of the experimental run). Eventually, for time $t=0$, the top side of the cell is heated to temperature $T_{2}$, while the bottom side is cooled to temperature $T_{1}$, resulting in a temperature difference $\Delta T=T_{2}-T_{1}$. Interference patterns are recorded to evaluate the phase difference variation as a function of time. This can be written as:

$$
\Delta \vartheta(t)=-\frac{2 \pi d^{*}}{\lambda_{0}} \Delta n(t)
$$

where $\Delta n$ represents the total variation of the refractive index between the hot and cold sides of the cell. For binary systems, the variation of refractive index after applying a temperature gradient is:

$$
\Delta n=\frac{\partial n}{\partial T} \Delta T+\frac{\partial n}{\partial c} \Delta c
$$

where $\Delta c$ is the concentration difference of the densest component between the hot and the cold sides of the cell. Coefficients $\partial n / \partial T$ and $\partial n / \partial c$ are the so-called contrast factors [13-20]. At the initial stage, the thermal kinetics provides a significant change in the magnitude of the phase difference. On the contrary, in a second stage the concentration kinetics becomes dominant in generating phase difference change. Hence, the two effects are decoupled enough to clearly distinguish the solutal contribution from the temporal point of view [17-21].

The concentration difference that is measured after the establishment of the temperature gradient can be written using Eqs. (2) and (3) as:

$$
\Delta c=-\frac{\lambda_{0} \Delta \vartheta}{2 \pi d^{*} \frac{\partial n}{\partial c}}
$$

\section{Theoretical relations}

During the second stage, when the concentration variation becomes dominant, assuming no convection perturbs the process, the concentration difference of the densest component as a function of time is [20,21]:

$$
\Delta c=-S_{T} c_{0}\left(1-c_{0}\right) \Delta T\left[1-\frac{8}{\pi^{2}} \sum_{n, \text { odd }} \frac{1}{n^{2}} \exp \left(-n^{2} \frac{t}{\tau_{r}}\right)\right]
$$

The two unknown parameters in the above equation are the Soret coefficient $S_{T}$ and the relaxation time $\tau_{r}, c_{0}$ being the initial concentration of the densest component, and $t$ the time. The relaxation time, for diffusion in a porous medium, is given by [22]:

$$
\tau_{r}=\frac{L^{2}}{\pi^{2} D^{*}}
$$

where $L$ is the length of diffusion, which in our case can be identified with the distance between the central points of the two DVs where the laser beams pass ( $L=42 \mathrm{~mm}$ in our set-up). $D^{*}$ is the molecular diffusion coefficient in the porous medium, which is related to the molecular diffusion coefficient in the free liquid $D$ by the relation [22]:

$$
D^{*}=\frac{D}{\tau^{2}}
$$

where $\tau$ is the tortuosity of the porous medium.

From the phase difference between the two lasers beams $\Delta \vartheta^{\exp }$, we estimate the phase difference due to the concentration difference by using Eqs. (2) and (3):

$$
\Delta \vartheta^{\text {mass }}=\Delta \vartheta^{\exp }-\frac{2 \pi d^{*}}{\lambda_{0}} \frac{\partial n}{\partial T} \Delta T
$$

Then, the phase difference relative to the second kinetic is fitted via the following equation:

$$
\Delta \vartheta^{\text {fit }}(t)=A \times\left\{1-\frac{8}{\pi^{2}} \sum_{n, \text { odd }} \frac{1}{n^{2}} \exp \left(-n^{2} B t\right)\right\}
$$

The fitting procedure is made by means of least-squares method with $A$ and $B$ as free fitting parameters.

Comparing Eqs. (4), (5), and (9), one can relate the Soret coefficient to the fitting parameter $A$ as follows:

$$
S_{T}=\frac{A \times \lambda_{0}}{2 \pi d^{*} \frac{\partial n}{\partial c} c_{0}\left(1-c_{0}\right) \Delta T}
$$

On the other hand, by comparing Eqs. (5), (6), (7), one can relate the diffusion coefficient to the fitting parameter $B$ and the value of the tortuosity of the porous medium:

$$
D=\frac{B \times L^{2} \times \tau^{2}}{\pi^{2}}
$$


Table 1

Literature values for molecular diffusion $D$, Soret coefficient $S_{T}$ and contrast factors at $1 \mathrm{~atm}$ and $T=25^{\circ} \mathrm{C}$ for reference systems.

\begin{tabular}{lllll}
\hline System & $D \times 10^{-10}\left(\mathrm{~m}^{2} \mathrm{~s}^{-1}\right)$ & $S_{T} \times 10^{-3}\left(\mathrm{~K}^{-1}\right)$ & $(\partial n / \partial T)_{P, c} \times 10^{-4}\left(\mathrm{~K}^{-1}\right)$ & $(\partial n / \partial c)_{P, T}$ \\
\hline Tol+C6 [17] & 27.79 & 4.915 & -5.500 & \\
THN+C12 [23] & $6.21 \pm 0.06$ & $9.5 \pm 0.5$ & $-4.39 \pm 0.02$ & 0.1228 \\
\hline
\end{tabular}
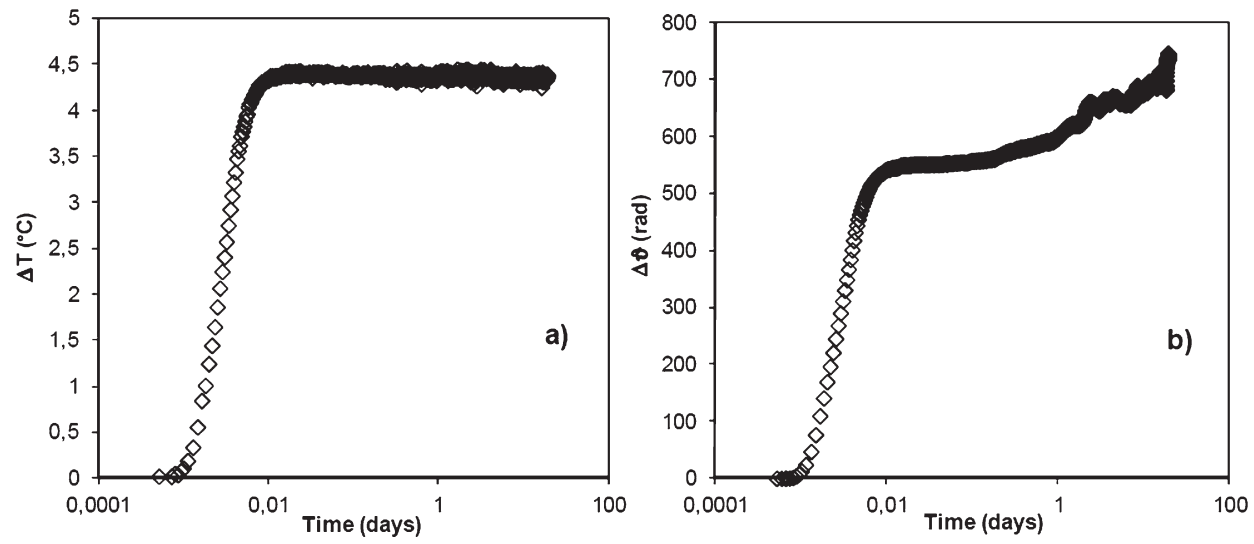

Fig. 1. a) Measured difference of temperature difference and b) phase difference between DVs versus time for Tol $+\mathrm{C} 6$ system $\left(c_{0}=51.7\right.$ wt $\%, T_{\text {mean }}=25^{\circ} \mathrm{C}$, at thermal steady state $\Delta T=4.4^{\circ} \mathrm{C}, p=0,1 \mathrm{MPa}$, porous medium).

Conversely, the value of the tortuosity can be obtained if the diffusion coefficient is known:

$$
\tau=\frac{\pi}{L} \sqrt{\frac{D}{B}}
$$

\section{Results and discussion}

\subsection{Validation of the cell at atmospheric pressure}

As a first step we have performed a series of experiments at atmospheric pressure by imposing a fixed temperature difference $\Delta T^{*}=10^{\circ} \mathrm{C}$ between the two thermostatic baths, with two well-characterized binary mixtures [17,23]. The mixtures are: the toluene + hexane system (in the following: Tol $+\mathrm{C} 6$ ), at the isomolar mass concentration $c_{0}=0.517$ of the first component, and 1,2,3,4-tetrahydronaphthalene $+n$-dodecane system (in the following: THN + C12), at mass fraction $c_{0}=0.5$. The mean temperature in the cell is set at $T_{\text {mean }}=25^{\circ} \mathrm{C}$. In Table 1 the reference values relevant for the cell validation are reported.

In Fig. 1a the temperature difference between the two DVs as a function of time is reported for the Tol+C6 system. As shown, the measured temperature difference $\Delta T$ between the two DVs is much smaller than the one applied to the two baths and it reaches the steady-state value of about $\Delta T=4.4^{\circ} \mathrm{C}$ due to a consistent loss of energy between the thermal baths and the cell.

In Fig. $1 \mathrm{~b}$ the phase difference between the beam that crossed the hot DV and the beam that crossed the cold one is plotted. It can be seen that while a fast variation is obtained due to the thermal effect (first kinetic) eventually a smaller and slower variation is observed due to the Soret-induced concentration gradient (second kinetic). As expected, the two kinetics are temporarily well separated.

For the Tol+C6 system the sign of the phase variation of the two kinetics is the same as one can expect if sample properties [17] and Eqs. (3) and (5) are considered:

$$
\Delta n=\frac{\partial n}{\partial T} \Delta T-\frac{\partial n}{\partial c} \times S_{T} \cdot c_{0}\left(1-c_{0}\right) \cdot \Delta T
$$

In fact, in this case, $\frac{\partial n}{\partial T}$ is negative, while $\frac{\partial n}{\partial c}$ and $S_{T}$ are positive, then both terms in the sum have the opposite sign with respect to the temperature gradient.

Little oscillations can be detected over the phase signal in Fig. 1b. Careful analysis of these oscillations indicates that they are mainly due to the ambient temperature oscillations (not shown). This effect can be filtered out from the raw data, by performing a de-convolution of the phase data with the ambient temperature ones.

Eventually the phase change of the second kinetics is considered. In Fig. 2 the second kinetics are reported for the two systems. The curves are shifted in order to start from the axes origin.

In the case of a porous medium, experiments can be nicely fitted with Eq. (9) thus meaning that experimental data points can be described by a 1D diffusive model. From the analysis in Fig. $2 \mathrm{~b}$ it is evident that data points in the free 

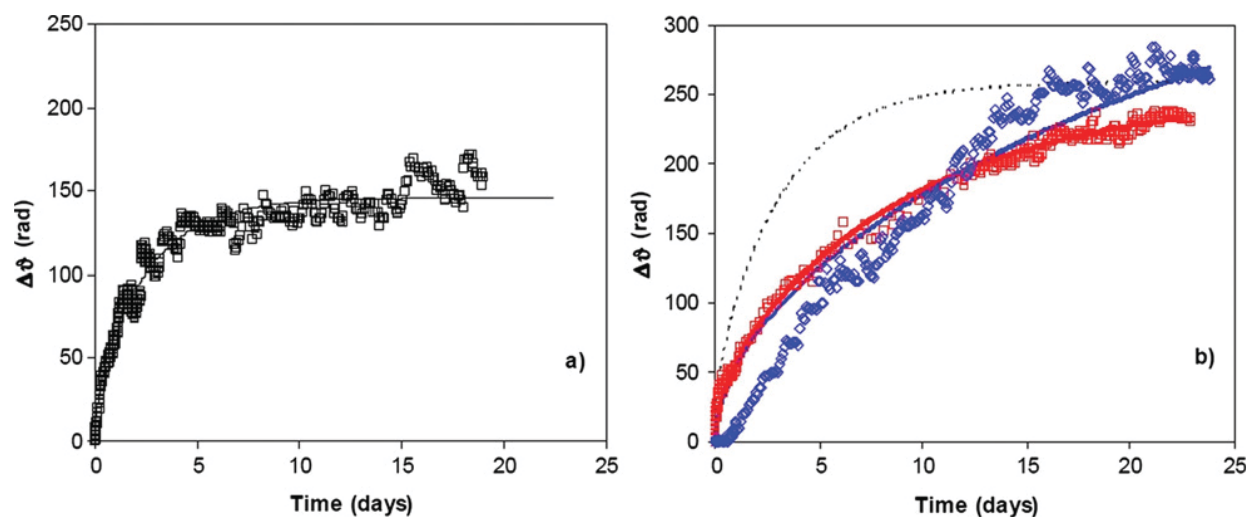

Fig. 2. Phase difference of the second kinetic versus time $\left(T_{\text {mean }}=25^{\circ} \mathrm{C}, \Delta T^{*}=10^{\circ} \mathrm{C}, p=0,1 \mathrm{MPa}\right)$. The continuous lines represent the fitting over the full experimental duration with 26 terms in Eq. (9). a) $\square$ represent the Tol+C6 system in porous medium $\left(c_{0}=51.7\right.$ wt\%). b) $\square$ represent THN+C12 system in porous medium $\left(c_{0}=50 \mathrm{wt} \%\right)$ and $\diamond$ in free medium. The dashed line represents the theoretical estimation given by Eqs. (4) and (5) with 26 terms in the sum and for diffusion in free medium.
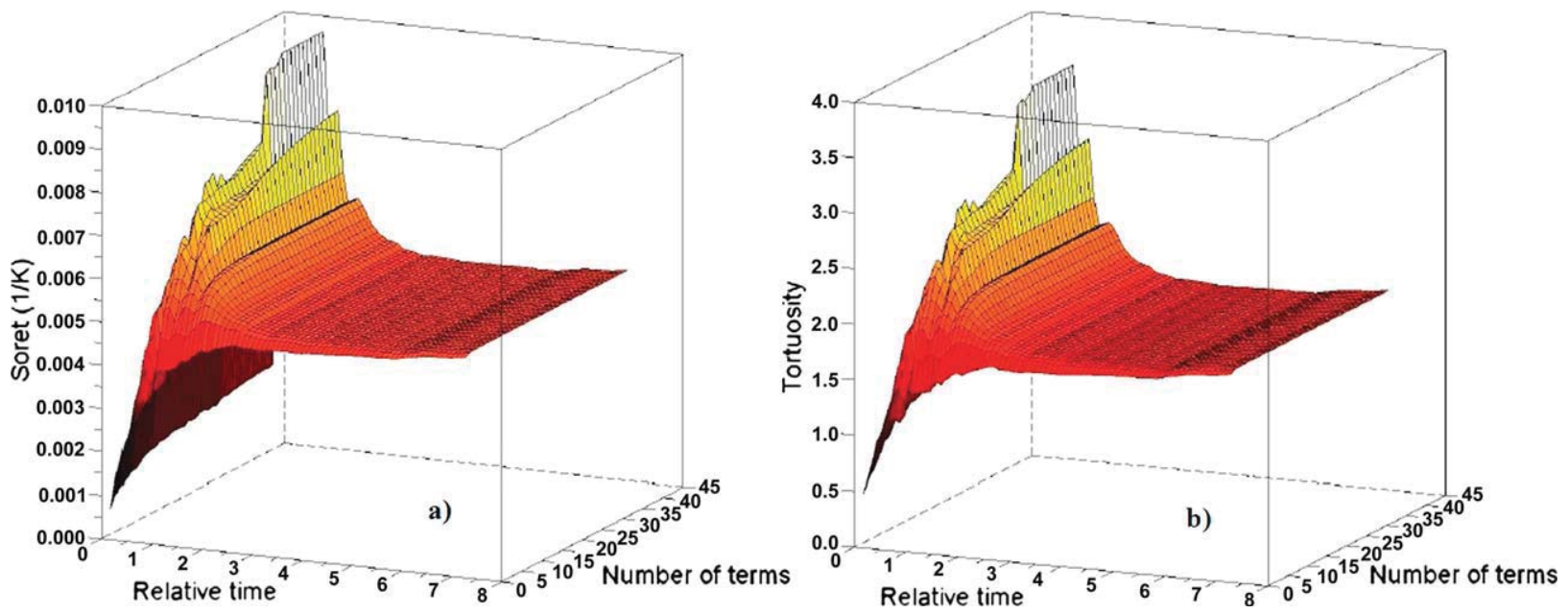

Fig. 3. a) Soret coefficient $S_{T}$, b) tortuosity $\tau$ of the porous medium as a function of the number of terms in the sum of Eq. (9) and of the relative time compared to the relaxation time for the Tol+C6 system $\left(T_{\text {mean }}=25^{\circ} \mathrm{C}\right.$, at thermal steady state $\Delta T=4.4^{\circ} \mathrm{C}, p=0,1 \mathrm{MPa}$, porous medium).

medium cannot be fitted through Eq. (9). The measured concentration gradient due to mass separation increases linearly and eventually saturates to a maximum value of separation. These data show that diffusion cannot be the only transport mechanism occurring in the cell in this case.

In Fig. 3 we report the obtained values of the Soret coefficients with Eq. (10) and the values of the tortuosity of the porous medium with Eq. (12) for Tol+C6 sample, as a function of the number of terms used in the sum of Eq. (9) and in function of the relative time compared to relaxation time.

For $S_{T}$ and $\tau$ the convergence is very fast as a function of the number of terms used in the sum, but only if the duration of the experiment is at least three times the relaxation time (five terms in the sums are enough). In Table 2 we report the values of the Soret coefficient $S_{T}$, its error compared to the reference value and the tortuosity $\tau$ of the porous medium as a function of the relative time for the Tol+C6 system. It is worth pointing out that for the evaluation of the Soret coefficient we have used the measured difference of temperature $\Delta T$ inside the cell at the thermal steady state. Moreover, for the evaluation of the tortuosity we have used Eq. (12), the values of the diffusion coefficients of Table 1 and $L=42 \mathrm{~mm}$ for the diffusion length.

Uncertainties indicated in the table are those due to the sensitivity of the least-squares fitting. After three times the relaxation time, the obtained agreement for the Soret coefficient is acceptable and after five times the relaxation time the obtained agreement is very good.

The obtained experimental value of the Soret coefficient of THN $+\mathrm{C} 12$ is $S_{T}=(9.3 \pm 0.2) \times 10^{-3} \mathrm{~K}^{-1}$, while the result for tortuosity is $\tau=1.78 \pm 0.06$. These values are coherent with the reference values of $S_{T}$ (see Table 1 ) and the tortuosity evaluated with Tol+C6 system. If the two values of the tortuosities obtained with the two systems are averaged, we get $\tau=1.81 \pm 0.15$ which standard deviation is about $8 \%$. This deviation can be considered acceptable if one takes into account the fact that the two systems are very different, for example their diffusion coefficients differ by about 63\% (see Table 1). 
Table 2

Soret coefficient $S_{T}$, deviation compared to the reference value and tortuosity $\tau$ of the porous medium for 26 terms in Eq. (9) in function of the relative time for the Tol $+\mathrm{C} 6$ system $\left(T_{\text {mean }}=25^{\circ} \mathrm{C}\right.$, at thermal steady state $\left.\Delta T=4.4^{\circ} \mathrm{C}, p=0,1 \mathrm{MPa}\right)$.

\begin{tabular}{llll}
\hline Time & $S_{T} \times 10^{-3}\left(\mathrm{~K}^{-1}\right)$ & Deviation & $\tau$ \\
\hline $2 \times \tau_{r}$ & $7 \pm 1$ & $+42 \%$ & $2.7 \pm 0.5$ \\
$3 \times \tau_{r}$ & $4.8 \pm 0.2$ & $-2 \%$ & $1.8 \pm 0.1$ \\
$4 \times \tau_{r}$ & $4.83 \pm 0.13$ & $-2 \%$ & $1.83 \pm 0.09$ \\
$5 \times \tau_{r}$ & $4.83 \pm 0.13$ & $-2 \%$ & $1.83 \pm 0.09$ \\
$6 \times \tau_{r}$ & $4.92 \pm 0.13$ & $+0.1 \%$ & $1.88 \pm 0.09$ \\
$7.26 \times \tau_{r}$ & $4.92 \pm 0.13$ & $+0.1 \%$ & $1.88 \pm 0.09$ \\
\hline
\end{tabular}

Table 3

Porosity $\varepsilon$, permeability $K$ and tortuosity $\tau$ measured by mercury porosimetry as a function of the cycles of intrusion and extrusion.

\begin{tabular}{lccc}
\hline Cycle & 1 & 2 & 3 \\
\hline$\varepsilon(\%)$ & 64.9 & 63.1 & 62.5 \\
$K(\mathrm{Da})$ & 268 & 262 & 260 \\
Tortuosity $\tau$ & 1.72 & 1.74 & 1.75 \\
\hline
\end{tabular}

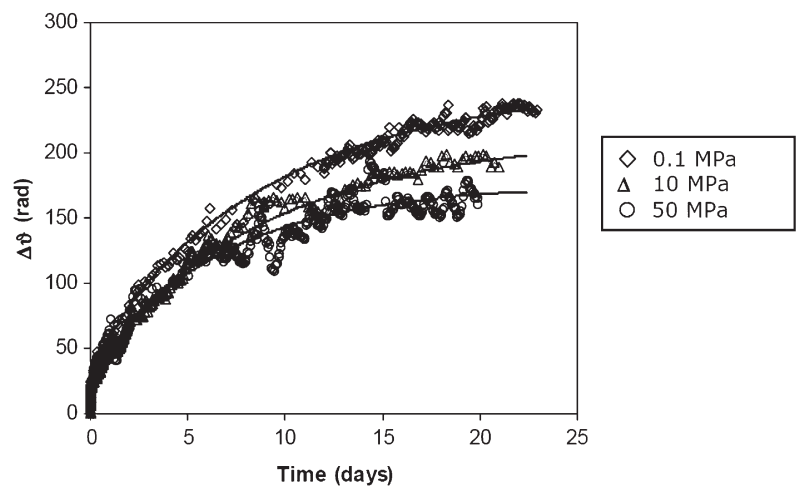

Fig. 4. Phase difference versus time of the second kinetic for the $\mathrm{THN}+\mathrm{C} 12$ system at different pressures.

After the series of experiments at atmospheric pressure and at high pressure, the porous medium was removed from the cell and analyzed by gas porosimetry (nitrogen) with a Tristar instrument from Micromeritics. The specific surface was evaluated at $266.93 \mathrm{~m}^{2} / \mathrm{g}$. Eventually, mercury porosimetry was performed with an Autopore also from Micromeritics, based on the capillary law governing mercury penetration into the pores [24]. In one cycle of this measurement, the pressure is increased from atmospheric pressure until $200 \mathrm{MPa}$ and after decreased. The results of three intrusion/extrusion cycles are reported in Table 3.

The value of the tortuosity is in good agreement with the mean value of the tortuosity measured with the thermodiffusion experiment.

\subsection{High-pressure measurements}

A series of measurements have been performed at high pressure with the THN+C12 system $\left(50 \% \mathrm{w} / \mathrm{w}, T=25^{\circ} \mathrm{C}, \Delta T^{*}=\right.$ $10^{\circ} \mathrm{C}$ ) before performing the porosimetry analysis. In Fig. 4 we report the phase difference versus time of the second kinetic for the $\mathrm{THN}+\mathrm{C} 12$ system at different pressures.

In Table 4 we report the relative duration of the experiment, mass contrast factor $\partial n / \partial c$, the Soret coefficient $S_{T}$, molecular diffusion coefficients in porous medium and in free medium $D^{*}$ and $D$, tortuosity $\tau$ and thermodiffusion coefficient $D_{T}$ for the system THN+C12 at different pressures.

The mass contrast factors $\partial n / \partial c$ have been measured in a precedent work [13]. From fitting experimental points we have determined the Soret and the diffusion coefficients in porous medium $S_{T}$ and $D^{*}$. By using a Leffler-Cullinan relation [25], it is also possible to get an evaluation of the molecular diffusion coefficient in free media $D$ as a function of the pressure:

$$
D \times \mu \approx \text { constant }
$$

where $\mu$ is the dynamic viscosity of the fluid. For the THN+C12 system, the viscosity has been measured from atmospheric pressure until $14 \mathrm{MPa}$ with a falling-body semi-automatic Stony Brook Scientific HPHTV-100 viscometer and with a falling-body viscometer developed in our laboratory [26] from atmospheric pressure until $50 \mathrm{MPa}$. Those measurements are reported in Fig. 5. 
Table 4

Relative duration of the experiment, Mass $\mathrm{CF} \partial n / \partial c$, Soret coefficient $S_{T}$, molecular diffusion coefficients in porous medium and in free medium $D^{*}$ and $D$, tortuosity $\tau$ and thermodiffusion coefficient $D_{T}$ for the system THN+C12 at different pressures.

\begin{tabular}{clllllll}
\hline $\begin{array}{l}\text { Pressure } \\
(\mathrm{MPa})\end{array}$ & Duration & $(\partial n / \partial c)_{P, T}$ & $\begin{array}{l}S_{T} \times 10^{-3} \\
\left(\mathrm{~K}^{-1}\right)\end{array}$ & $\begin{array}{l}D^{*} \times 10^{-10} \\
\left(\mathrm{~m}^{2} \mathrm{~s}^{-1}\right)\end{array}$ & $\begin{array}{l}D \times 10^{-10} \\
\left(\mathrm{~m}^{2} \mathrm{~s}^{-1}\right)\end{array}$ & $\begin{array}{c}\tau \\
D_{T} \times 10^{-12} \\
\left(\mathrm{~m}^{2} / \mathrm{s} \mathrm{K}\right)\end{array}$ \\
\hline 0.1 & $2.1 \times \tau_{r}$ & 0.1170 & $9.3 \pm 0.2$ & - & $6.219^{\mathrm{a}}$ & 1.78 & 5.78 \\
10 & $2.1 \times \tau_{r}$ & 0.1153 & $8.1 \pm 0.3$ & $2.1 \pm 0.2$ & 5.42 & 1.60 & 4.39 \\
50 & $3 \times \tau_{r}$ & 0.1140 & $6.6 \pm 0.2$ & $3.1 \pm 0.2$ & 3.66 & 1.09 & 2.42 \\
\hline
\end{tabular}

a Reference value.

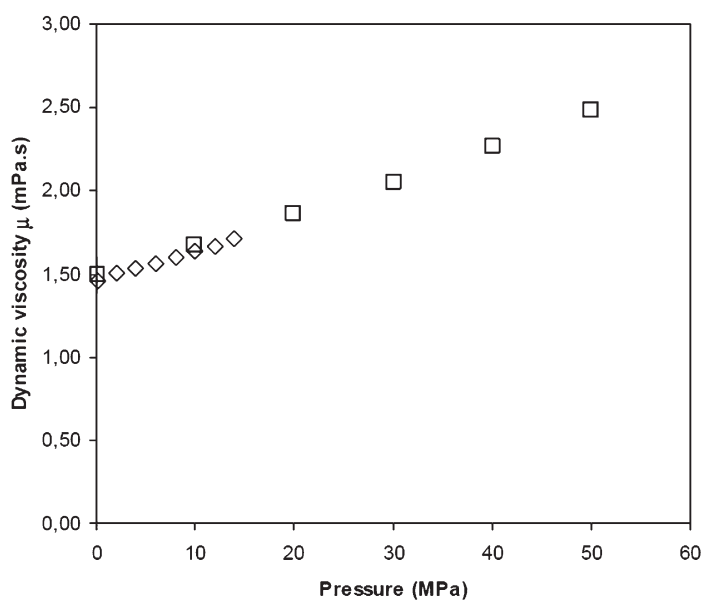

Fig. 5. Dynamic viscosity as a function of the pressure of the $\mathrm{THN}+\mathrm{C} 12$ system $\left(T_{\text {mean }}=25^{\circ} \mathrm{C}, c_{0}=50\right.$ wt $\%$ ). Diamonds represent measurements made with the Stony Brook viscometer and squares represent measurements made with the isobar transfer viscometer.

At atmospheric pressure and with the literature value for $D$, we evaluate the constant in Eq. (14). In Table 4 we report the evaluated values of diffusion coefficient in free media $D$ thanks to the measured values of viscosity. Also in Table 4 we report the evaluation of the tortuosity of the porous medium by using Eq. (7). Thermodiffusion coefficients are evaluated using:

$$
D_{T}=S_{T} \times D
$$

The last column of Table 4 represents the relative deviation from reference $D_{T}$ values [10]. The measurements at $50 \mathrm{MPa}$ were not performed with the thermogravitational technique. The agreement is good, taking into account the experimental errors and our hypothesis in the evaluation of the diffusion coefficients $D$ at high pressure. We observe a decrease of the value of the Soret coefficient $S_{T}$ as a function of the pressure. Also the tortuosity $\tau$ decreases as a function of pressure. Further analysis is needed to understand this counterintuitive behaviour in function of pressure.

\section{Conclusions}

In this work we have demonstrated the functionality of our thermodiffusion cell designed to measure molecular diffusion and Soret coefficients for binary systems under high pressure and in porous medium. With the cell filled with a porous medium, the main mass transport mechanism is diffusion. This is not the case when the cell is free of porous medium, probably due to residual convection.

From the analysis of the second kinetic of the phase difference between the two laser beams crossing the cell, we recover values of the Soret coefficient of the analyzed mixtures in good agreement with literature data. The value of the tortuosity of the porous medium could also be evaluated from the transient state of the mass separation. For the mixture of toluene + hexane and 1,2,3,4-tetrahydronaphthalene + dodecane, we obtain a mean value of the tortuosity at atmospheric pressure of 1.81. This value is in good agreement with the value obtained with a standard technique for characterizing porous media, namely the mercury porosimetry.

Preliminary results at high pressure show a decrease of the Soret coefficient of the 1,2,3,4-tetrahydronaphthalene + dodecane system and a decrease of the porous medium tortuosity at high pressure.

\section{Acknowledgements}

This work was financially supported under ESA contract 14228/00/NL/SH (DSC-SCCO3 project) and the PRES of Bordeaux. H.B. wishes to thank J.-L. Daridon of the LFC-R at UPPA for proposing the research subject and introducing himself into 
the DSC-SCCO3 project, V. Shevtsova, J.-C. Legros, A. Mialdun, and D. Melnikov of the MRC at ULB for valuable discussions. F.C. acknowledges present support from the European Union under a Marie Curie funding, contract No. IEF-251131, DyNeFI Project. Authors are grateful to J.-P. Bazille and B. Guatarbès for the help in viscosity measurements and porous medium characterization.

\section{References}

[1] C. Ludwig, Sitzungsber. Preuss. Akad. Wiss., Phys. Math. Kl. 20 (1856) 539.

[2] C. Soret, Arch. Sci. Phys. Nat. 3 (1879) 48;

C. Soret, C. R. Acad. Sci. III 91 (1880) 289.

[3] W. Köhler, S. Wiegand, Thermal Non-equilibrium Phenomena in Fluid Mixtures, Springer, Berlin, 2002.

[4] S.R. de Groot, P. Mazur, Non-equilibrium Thermodynamics, Dover, New York, 1984.

[5] S. Van Vaerenbergh, A. Shapiro, G. Galliero, F. Montel, J.-C. Legros, J. Caltagirone, J.-L. Daridon, M.Z. Saghir, Multicomponent processes in crudes, in: European Space Agency Special Publication, vol. 1290, ESA Communications Production, Noordwijk, the Netherlands, 2005, pp. 202-213.

[6] K. Ghorayeb, A. Firoozabadi, T. Anraku, SPE J. 8 (2003) 114.

[7] F. Montel, J. Bickert, A. Lagisquet, G. Galliero, J. Petrol. Sci. Eng. 58 (2007) 391.

[8] W.M. Rutherford, H.G. Drickamer, J. Chem. Phys. 22 (1954) 1157.

[9] W.M. Rutherford, J.G. Roof, J. Phys. Chem. 63 (1959) 1506.

[10] P. Urteaga, M.M. Bou-Ali, D. Alonso de Mezquia, J. Santamaría, C. Santamaría, J. Madariaga, H. Bataller, Rev. Sci. Instrum. 83 (2012) 074903.

[11] S. Van Vaerenbergh, S. Srinivasan, M.Z. Saghir, J. Chem. Phys. 131 (2009) 114505-1.

[12] M. Touzet, G. Galliero, V. Lazzeri, Z. Saghir, F. Montel, J.-C. Legros, C. R. Méc. 339 (2011) 318.

[13] F. Croccolo, M.-A. Arnaud, D. Bégué, H. Bataller, J. Chem. Phys. 135 (2011) 034901.

[14] F. Croccolo, F. Plantier, G. Galliero, G. Pijaudier-Cabot, M.Z. Saghir, F. Dubois, S. Van Vaerenbergh, F. Montel, H. Bataller, Rev. Sci. Instrum. 82 (2011) 126105.

[15] F. Croccolo, H. Bataller, G. Pijaudier-Cabot, AIP Conf. Proc. 1254 (2010) 157.

[16] A. Becker, W. Köhler, B. Muller, Ber. Bunsenges. Phys. Chem. 99 (1995) 600

[17] K.J. Zhang, M.E. Briggs, R.W. Gammon, J.V. Sengers, J. Chem. Phys. 104 (1996) 6881.

[18] R. Kita, S. Wiegand, J. Luettmer-Strathmann, J. Chem. Phys. 121 (2004) 3874.

[19] G. Wittko, W. Köhler, Phil. Mag. 83 (2003) 1973.

[20] A. Mialdun, V.M. Shevtsova, Int. J. Heat Mass Transfer 51 (2008) 3164.

[21] A. Mialdun, V.M. Shevtsova, J. Chem. Phys. 134 (2011) 044524.

[22] C. Jiang, T. Jaber, H. Bataller, M.Z. Saghir, Int. J. Thermal Sci. 47 (2008) 126.

[23] J.K. Platten, M.M. Bou-Ali, P. Costesèque, J.-F. Dutrieux, W. Köhler, C. Leppla, S. Wiegand, G. Wittko, Phil. Mag. 83 (2003) 1965.

[24] C.A. León y León, Adv. Colloid Interface Sci. 76-77 (1998) 341.

[25] J. Leffler, H.T. Cullinan Jr., Ind. Eng. Chem. Fundam. 9 (1970) 84.

[26] P. Daugé, A. Baylaucq, L. Marlin, C. Boned, J. Chem. Eng. Data 46 (2001) 823. 